\title{
Markov Chain Approximation method for Pricing Barrier Options with Stochastic Volatility and Jump
}

\author{
Sumei Zhang \\ Department of applied mathematics \\ School of science, Xi' an university of Post and Telecommunications \\ Xi' an, China \\ zhanggsumei@sina.com
}

\begin{abstract}
The purpose of this paper is to provide an efficient pricing method for barrier option with stochastic volatility and jump risk. First, by constructing a nonuniform variance grid and using local consistency arguments, this paper approximates the stochastic volatility jump-diffusion model with a finite and dense Markov chain; Then, the paper computes the rate matrix of the Markov chain by solving a system induced by local consistency conditions; And then the paper provides the character function of the Markov chain. At last, using Markov chain approximation method and Fourier transform technique, the paper obtains numerical solutions for barrier options pricing. Numerical results show that comparing with the Monte Carlo simulation, the proposed pricing technique is accurate, fast and easy to implement.
\end{abstract}

Keywords-Barrier option; option pricing; Markon chain; stochastic volatility; jump diffusion

\section{INTRODUCTION}

Barrier options [1] are among the most popular pathdependent derivatives that disappear or appear if the underlying asset price crosses a given level (barrier level) before expiration date. Such contracts form effective risk management tools, and are liquidly traded in the foreign exchange markets. The most frequently used standard barrier options are knock in and knock out options. Knock in options can be divided into two categories, up and in, and down and in. Similarly, knocking out options can also be used as a down and out and up and out option. This paper focuses on standard knock out call options.

There exists currently a good deal of literature on numerical methods for the pricing of barrier options. It is well known that in this case a straightforward Monte Carlo simulation algorithm will be time-consuming and yield unstable results for the prices and especially the sensitivities. Since option pricing can be described as the solution of a partial differential equation (PDE) or partial integrodifferential equation (PIDE) with boundary condition [2], some researchers have priced barrier options through PDE (PIDE) method under stochastic volatility model or jumpdiffusion model [3-7]. However some empirical evidences
[8-9] show that the model that combines stochastic volatility and jumps may be more reasonable. But a complex model with too many stochastic factors will lead to difficulty of obtaining the solution of the corresponding pricing equation.

A different approach, pioneered by Kushner [10], is the Markov chain approximation method. Originally developed for the numerical solution of stochastic optimal control problems in continuous time, this method consists of approximating the system of interest by a discrete time chain that closely follows its dynamics, and solving the problem of interest for this chain. An application to the pricing of American options under jump diffusion model is given in [11]. Zhang et al [12] consider lookback options pricing in a stochastic volatility model. Mijatović [13] prices barrier options in a local volatility jump diffusion model. However, there is rare study for valuation of barrier option under a stochastic volatility jump diffusion model, which is rather challenge due to the nonlinearity and jump discontinuity.

The rest of the paper is organized as follows. Section 2 develops the underlying pricing model. Section 3 describes Markon chain approximation method. Section 4 presents numerical results for barrier options pricing. Section 5 concludes the paper.

\section{THE MODEL}

An arbitrage-free, frictionless financial market is considered where only riskless asset $B$ and risky asset $S$ are traded continuously up to a fixed horizon date $T$. Let $\left(\Omega, \mathcal{F}, \mathcal{F}_{t}, P\right)$ be a complete probability space with a filtration satisfying the usual conditions, i.e. the filtration is continuous on the right. Suppose $W^{1}(t)$ and $W^{2}(t)$ are both standard Brownian motion which is $\mathcal{F}_{t}$-adapted, and $W^{1}(t)$ has correlation $\rho$ with $W^{2}(t)$.Let $N(t)$ be independent Poisson process with constant intensity $\lambda, V(t)$ and $S(t)$ denote the volatility and price process of stock. According to [14], the stochastic volatility jump-diffusion 
model can be represented by the following:

$$
\left\{\begin{array}{l}
d \log S(t)=(r-\lambda \bar{\mu}) d t+\sqrt{V(t)} d W^{1}(t)+\xi_{s} d N(t) \\
d V(t)=k(\theta-V(t)) d t+\sigma \sqrt{V(t)} d W^{2}(t) \\
d W^{1}(t) W^{2}(t)=\rho d t
\end{array}\right.
$$

where $r$ is constant interest rate, $\xi_{s}$ is the jump size, and $\ln \left(\xi_{s}+1\right)$ has an normal distribution with mean $\mu_{J}$ and variance $\sigma_{J}^{2} \cdot \lambda \bar{\mu}$ denotes the drift term compensates for the expected drift added by the jump component. By the Itô formula, $\bar{\mu}=E\left(\xi_{S}\right)=\exp \left(\mu_{J}+\frac{1}{2} \sigma_{J}{ }^{2}\right)-1 . V(t)$ is a square root mean reverting process, first proposed by Heston [15]. $k, \theta, \sigma$ The parameter $k>0$ is the mean-reversion rate, $\theta>0$ is the longterm mean, $\sigma>0$ is the volatility-ofvariance. In this paper, the author always assumes that $2 k \theta>\sigma^{2}$, which is known as the Feller condition.

\section{OPTION PRICING BASE D ON MARKON CHAIN APPROXIMATION METHOD}

According to [16], the volatility process $V(t)$ can be approximated arbitrarily well using a carefully selected Markov chain which satisfies the local consistency requirements.

A. The "local consistency" concept

Suppose $V^{h}=\left\{V_{0}{ }^{h}, V_{1}{ }^{h}, \cdots, V_{N_{h}}{ }^{h}\right\}$ is a variance grid where $h>0$ denotes the spacing between discrete points. Assume that $\left|V^{h}(t+\delta)-V^{h}(t)\right| \rightarrow 0, N_{h} \rightarrow \infty$ and the grid can cover the domain of $V(t)$ as $h \rightarrow 0$. Suppose $V^{h}(t) \in V$ denote the approximating chain of the process $V(t)$, and the corresponding rate matrix is $Q^{h}=\left[q_{i j}^{h}\right]$.

Assume that the values of the variance process and the approximating chain coincid, $V^{h}(t)=V(t)$. Suppose $E_{t}^{h}$ denotes the expectation. For some $\delta>0, V^{h}(t)$ meets the following local consistency conditions [10]:

$$
E_{t}^{h}\left\{V^{h}(t+\delta)-V^{h}(t)\right\}=k(\theta-V(t)) \delta+o(\delta)
$$

$$
\begin{gathered}
E_{t}^{h}\left\{V^{h}(t+\delta)-V^{h}(t)\right\}^{2}=\sigma^{2} V(t) \delta+o(\delta) \\
\left|V^{h}(t+\delta)-V^{h}(t)\right|=o(h)
\end{gathered}
$$

\section{B. Construction of the approximating Markov chain of the model}

1) The grid

A suitable choice of the grid is essential for the effectiveness of the pricing algorithm. One of the features of a good grid is that it has sufficient resolution in regions of interest, such as the current spot value and the barrier levels, which is a necessary condition for constructing a Markov chain market model that approximates well the dynamics of the given price process. Another desirable feature is that the grid "covers" a sufficiently large part of the state-space, which is needed to control the truncation error that arises when approximating an infinite state-space by a finite statespace. To employ a uniform grid that satisfies these conditions would be computationally expensive. Here the author employs the following procedure for generating a suitable nonuniform grid $\mathrm{G}$, based on an algorithm from [13]:

a) Pick $N_{i} \in N$ and $d_{i}^{ \pm} \in(0,+\infty), i=1,2,3$ and the smallest and largest values $V_{1}, V_{N}$ of the grid $V$, such that $N=N_{1}+N_{2}+N_{3}$.

b) Define the subgrid

$$
G_{i}=G\left(a_{i}, s_{i}, b_{i}, N_{i}, d_{i}^{-}, d_{i}^{+}\right), i=1,2,3 .
$$

where $a_{1}=V_{1}, s_{1}=l, s_{2}=S_{0}$, $s_{3}=u, b_{1}=a_{2}, b_{2}=a_{3}, b_{3}=V_{N}$.

The subgrid $G_{i}$ is generated by the following procedure:

- Compute $c_{1}=\arcsin h\left(\frac{a-s}{g_{1}}\right), c_{2}=\arcsin h\left(\frac{b-s}{g_{2}}\right)$.

- Define the lower part of the grid by the formula

$$
x_{k}=s+g_{1} \sinh \left(c_{1}(1-(k-1) /(M / 2-1))\right) \text {, }
$$

where $k \in\{1, \ldots, M / 2\}$.

- Define the upper part of the grid by the formula

$$
x_{k+M / 2}=s+g_{2} \sinh \left(c_{2} 2 k / M\right)
$$

where $k \in\{1, \ldots, M / 2\}$.

c) $G=G_{1}+G_{2}+G_{3}$.

2) Computation of the rate matrix

Assume that at time t, the variance is equal to $V_{j}^{h}$. Over a time interval $\delta$, there are three possibilities: remain at $V_{j}^{h}$, move up by $d_{U}$ to $V_{j+1}^{h}=V_{j}+d_{U}$, or move down by $d_{D}$ to $V_{j-1}^{h}=V_{j}-d_{D}$. Local consistency condition (2) and (3) can therefore be restated as

$$
-q_{j, j-1}^{h} d_{D} \delta+q_{j, j+1}^{h} d_{U} \delta=(r-\lambda \bar{\mu}) \delta+o(\delta)
$$

$$
q_{j, j-1}^{h} d_{D}^{2} \delta+q_{j, j+1}^{h} d_{U}^{2} \delta=V^{h}(t) \delta+o(\delta)
$$

(6)

Solving the above system, it can be obtained

$$
\begin{gathered}
q_{j, j-1}^{h}=\frac{\sigma^{2}}{2 h^{2}} \exp \left(-V_{j}^{h}\right)-\frac{k}{h}\left(\left(\theta-\frac{\sigma^{2}}{2 k}\right) \exp \left(-V_{j}^{h}\right)-1\right), \\
q_{j, j}^{h}=-\frac{\sigma^{2}}{h^{2}} \exp \left(-V_{j}^{h}\right), \\
q_{j, j+1}^{h}=\frac{\sigma^{2}}{2 h^{2}} \exp \left(-V_{j}^{h}\right)+\frac{k}{h}\left(\left(\theta-\frac{\sigma^{2}}{2 k}\right) \exp \left(-V_{j}^{h}\right)-1\right) .
\end{gathered}
$$

3) The characteristic function 
Using the decompositions $W^{1}=\rho W^{2}+\sqrt{1-\rho^{2}} Z$, where $Z$ is a standard Brownian motion, independent of all other processes, Model (1) can be written as

$$
\begin{aligned}
d \log S(t)= & (r-\lambda \bar{\mu}) d t+\sqrt{V(t)} \rho d W^{2}(t)+\sqrt{V(t)} \sqrt{1-\rho^{2}} d Z \\
& +\xi_{s} d N(t)
\end{aligned}
$$

Equation (8) implies that $d W^{2}=\frac{d V(t)-k(\theta-V(t)) d t}{\sigma \sqrt{V(t)}}$, which can be substituted into the first expression, and substitute $V(t)$ by $V^{h}(t)$, it can be obtained

$$
\begin{aligned}
d \log S(t) \approx & (r-\lambda \bar{\mu}) d t+\frac{\rho}{\sigma}\left(d V^{h}(t)-k\left(\theta-V^{h}(t)\right) d t\right) \\
& +\sqrt{V(t)} \sqrt{1-\rho^{2}} d Z+\xi_{s} d N(t) .
\end{aligned}
$$

As [16] shows, the approximating characteristic function of $\log \frac{S(t)}{S(0)}$ is given by

$$
\phi_{i}(u)=l^{T} \exp \{t \mathrm{~B}(\mathrm{u})\} e_{i}
$$

where $l$ is an $(\mathrm{n} \times 1)$ vector of ones, and $e_{i}$ is the $i$-th $(\mathrm{n} \times 1)$ unit vector. The matrix function $\mathrm{B}(\mathrm{u})$ has elements of the form:

$$
\beta_{j, i}= \begin{cases}q_{j, j}^{h}+\Psi_{j}^{h}(u), & i=j \\ q_{j, i}^{h}+\Psi_{j \pm}^{h}(u), & i=j \pm 1 \\ 0, & \text { otherwise. }\end{cases}
$$

where $\Psi_{j}^{h}(u)=i(r-\lambda \bar{\mu}) u-\frac{1}{2}\left(1-\rho^{2}\right) V^{h}(t) u^{2}+\lambda\left(\phi_{J}-1\right)$, $\phi_{J}=\exp \left\{-\lambda \bar{\mu} i u t+\lambda t\left[(1+\bar{\mu})^{i u} \exp \left(\frac{1}{2} \sigma_{J}^{2} i u(i u-1)\right)-1\right]\right\}$, $\Psi_{j \pm}^{h}(u)=\exp \left\{ \pm i \frac{\rho}{\sigma} h u\right\}$.

\section{Barrier option pricing}

Let $\log S=x$ and denote with $U(x, t)$ the value of the barrier option at time $t$. $U(x, t)$ can be computed by

$$
U(x, t)=E\left[C\left(S\left(\tau_{B}\right), \tau_{B}\right)\right]
$$

where $C\left(S\left(\tau_{B}\right), \tau_{B}\right)$ is a discounted payoff function and $\tau_{B}$ is the first hitting time of the given barrier level $B$ by the underlying asset process $S(t)$. For down-and-out call barrier options the payoff $C\left(S\left(\tau_{B}\right), \tau_{B}\right)$ is defined by

$$
C\left(S\left(\tau_{B}\right), \tau_{B}\right)= \begin{cases}e^{-r T} \max (S(T)-K, 0), & \tau_{B}=T, \\ 0, & \tau_{B}<T .\end{cases}
$$

where $K$ is given exercise price at expiration date $T$.
Using Markon chain approximation method, $U(x, t)$ can be obtain by the recursive relationship

$$
\begin{aligned}
U(x, t) \approx & \exp (-r \Delta t) E\left(U\left(X_{t+\Delta t}, t+\Delta t, s_{t+\Delta t}\right) \mid X_{t}=x, s_{t}=i\right) \\
= & \exp (-r \Delta t) \sum_{j=1}^{N} \int_{R} U(y, t+\Delta t, j) \\
& \times \mathrm{P}\left(X_{t+\Delta t}-x \in d y \mid s_{t}=i, s_{t+\Delta t}=j\right) \mathrm{P}\left(s_{t+\Delta t}=j \mid s_{t}=i\right)
\end{aligned}
$$

If denote with $f(y)$ the log-return density over a time interval of $\Delta t$, then the above relationship can be written as

$$
U(x, t, i)=\exp (-r \Delta t) \sum_{j=1}^{N} \int_{R} U(y, t+\Delta t, j) f(y-x, i, j) .
$$

$f(y, i, j)$ can be retrieved by taking the inverse Fourier transform of the characteristic function $\phi_{i}(u)$.

\section{NUMERICAL EXAMPLE}

This section uses the method from Section 3 to price barrier options. This paper first evaluates barrier options using Markov chain approximation method. Then a comparison of the speed and accuracy between the Markov chain approximation and the exact Monte Carlo simulation proposed by [17] is provided. For comparison the default parameters are used in [17] and listed in Table I .

TABLE I. DEFAULT PARAMETERS FOR BARRIER OPTIONS PRICING

\begin{tabular}{|c|c|}
\hline Parameter & Value \\
\hline Initial asset price & $S(0)=100$ \\
\hline Intensity of the Poisson process & $\lambda=0.11$ \\
\hline Volatility of volatility & $\sigma=0.27$ \\
\hline Interest rate & $r=0.0319$ \\
\hline Long-run variance & $\theta=0.014$ \\
\hline Initial variance & $V(0)=0.008836$ \\
\hline Mean reversion & $k=3.99$ \\
\hline expectation of the jump size & $\bar{\mu}=-0.12$ \\
\hline $\begin{array}{c}\text { Correlation between returns and } \\
\text { volatility }\end{array}$ & $\rho=-0.79$ \\
\hline Maturity date & $T=5$ \\
\hline
\end{tabular}

Table II lists the comparison of the two methods. Except for barrier level $B=90$, other parameters are the same as the ones in Table 1. For Monte Carlo, number of simulation is 100000 . For Markov chain approximation, number of grid is 200 . 
TABLE II. COMPARISON OF DOWN AND OUT CALL OPTIONS PRICES BETWEEN MARKOV CHAIN APPROXIMATION AND MONTE CARLO SIMULATION

\begin{tabular}{|c|c|c|}
\hline $\begin{array}{c}\text { Exercise } \\
\text { price }\end{array}$ & $\begin{array}{c}\text { Exact Monte Carlo } \\
\text { simulation }\end{array}$ & $\begin{array}{c}\text { Markov chain } \\
\text { approximation }\end{array}$ \\
\hline 80 & $32.3423(0.0284)$ & 32.3417 \\
\hline 85 & $28.9055(0.0271)$ & 28.9060 \\
\hline 90 & $25.4688(0.0259)$ & 25.4679 \\
\hline 95 & $22.1107(0.0248)$ & 22.1099 \\
\hline 100 & $18.9709(0.0235)$ & 18.9701 \\
\hline 105 & $16.0827(0.0220)$ & 16.0833 \\
\hline 110 & $13.4641(0.0204)$ & 13.4633 \\
\hline 115 & $11.1232(0.0188)$ & 11.1225 \\
\hline 120 & $9.0646(0.0171)$ & 9.0651 \\
\hline
\end{tabular}

Note: Numbers in parentheses are standard errors for the estimates of options prices.

The numerical experiment shows that Markov chain approximation is considerably faster than the Monte Carlo simulation. For the pricing of down and out call options Markov chain approximation takes about 0.03 seconds, while Monte Carlo simulation takes about 9.1 seconds. Moreover, Table II suggests the accuracy of the Markov chain approximation method. If the Monte Carlo is considered to be the benchmark, the relative percentage pricing differences of Markov chain approximation are all less than $0.09 \%$.

\section{CONCLUSION}

This paper combines stock price jumps and stochastic volatility and considers a general jump-diffusion model for pricing barrier options. By Markov chain approximation method and Fourier transform technique, the paper obtains numerical solutions for barrier options pricing. Numerical results show that the proposed pricing technique is accurate, fast and easy to implement. The paper presents an efficient pricing method for barrier option with stochastic volatility and jump risk.

\section{ACKNOWLEDGMENT}

This work is supported by the National Natural Science Foundation of China under Grant No.11426176, the Science Plan Foundation of the Education Bureau of Shaanxi Province under Grant No.14JK1672 and the Youth Foundation of Xi'an University of Post and Telecommunications under Grant No.ZL2013-33.

\section{REFERENCES}

[1] H. John, "Options, Futures, and Other Derivatives", 6th ed., Prentice Hall, 2005, pp.513-515.

[2] R. Cont and P. Tankov, "Financial Modelling with Jump Processes", Chapman and Hall/CRC Press, London, 2004.

[3] K. Oleg, "Finite Difference Methods for Option Pricing under Lévy Processes: Wiener-Hopf Factorization Approach", The Scientific World Journal, vol. 2013, pp. 963625-963625, 2013.

[4] I.Andrey and C. Peter, "Jumps Without Tears: A New Splitting Technology for Barrier Options", International Journal of Numerical Analysis and Modeling, vol. 8(4), pp. 667-704, 2011.

[5] J.C. Ndogmo and D.B. Ntwiga, "High-Order Accurate Implicit Methods for Barrier Option Pricing", Applied Mathematics and Computation, vol. 218(5), pp. 2210-2224, 2011.

[6] B. Luca Vincenzo and P. Graziella, "A Radial Basis Function Approach to Compute the First-Passage Probability Density Function in Two-Dimensional Jump-Diffusion Models for Financial and Other Applications", Engineering Analysis With Boundary Elements, vol. 36(11), pp. 1546-1554, 2012.

[7] H. Tinne, "Efficient and Stable Numerical Solution of the HestonCox-Ingersoll-Ross Partial Differential Equation by Alternating Direction Implicit Finite Difference Schemes", International Journal of Computer Mathematics, vol. 90(11), pp. 2409-2430, 2013.

[8] K. Larsson and M. Nossman, "Jumps and Stochastic Volatility in Oil Prices: Time Series Evidence", Energy Economics, vol. 33(3), pp. 504-514, 2011.

[9] H.G. Zhou and J. Zhu, "An Empirical Examination of Jump Risk in Asset Pricing and Volatility Forecasting in China's Equity and Bond Markets", Pacific-Basin Finance Journal, vol. 20(5), pp. 857-880, 2012.

[10] H.J. Kushner and P.G. Dupuis, "Numerical Methods for Stochastic Control Problems in Continuous Time", 2nd ed., New York: Springer, 2000

[11] J.G. Simonato, "Computing American Option Prices in the Lognormal Jump-Diffusion Framework with a Markov Chain", Finance Research Letters, vol. 8(4), pp. 220-226, 2011.

[12] Z.H. Zhang, G. Yin, and Z.A. Liang, "A Stochastic Approximation Algorithm for American Lookback Put Options", Stochastic Analysis and Applications, vol. 29(2), pp. 332-351, 2011.

[13] A. Mijatović, "Continuously Monitored Barrier Options under Markov Processes", Mathematical Finance, vol. 23(1), pp. 1-38, 2013.

[14] G. Bakshi, C. Cao, and Z. Chen, "Pricing and Hedging Long-Term Options", Journal of Ecnometrics, vol. 94(1-2), pp. 277-318, 2000.

[15] S.L. Heston, "A Closed-Form Solution for Options with Stochastic Volatility with Applications to Bond and Currency Options", Review of Financial Studies, vol. 6(2), pp. 327-343, 1993.

[16] K. Chourdakis, "Non-Affine Option Pricing", Journal of Derivatives, vol. 11 (3), pp. 10-25, 2004.

[17] M. Broadie, ö. Kaya, "Exact Simulation of Stochastic Volatility and Other Affine Jump Diffusion Processes", Operations Research, vol. 54(2), pp. 217-231, 2006. 